\title{
OS RISCOS ERGONÔMICOS NO COTIDIANO DAS EQUIPES DE ENFERMAGEM
}

\author{
THE ERGONOMIC RISKS IN THE DAILY TEAMS OF NURSING
}

\author{
B. B. de ANDRADE ${ }^{1}$, L. de F. SANTOS ${ }^{1}$ e L. M. TORRES ${ }^{1}$
}

${ }^{1}$ Faculdade Ciências Médicas de Minas Gerais. Curso de Enfermagem, Brasil.

\author{
ART ICLE INFO \\ Article history: \\ Received 2018-07-04 \\ Accepted 2018-10-15 \\ Available online 2018-10-31 \\ *Autor correspondente: \\ E-mail: beatrizbandrade@hotmail.com
}

Palavras-chave: Saúde do trabalhador. Engenharia humana. Riscos ocupacionais. Enfermagem.

Keywords: Worker Health. Human Engineering. Occupational Risks. Nursing.

RESUMO. O profissional ao desempenhar um trabalho se expõe aos riscos relacionados a ele, podendo adoecer física e mentalmente, devido às mudanças nos processos de trabalho, associadas ao ritmo intenso e longas jornadas. Os trabalhadores da equipe de enfermagem, especificamente, prestam assistência direta ao indivíduo, em longos períodos de tempo, o que aumenta a exposição aos riscos ocupacionais. Revisão integrativa de literatura nacional e internacional cujo objetivo foi identificar os riscos ergonômicos no cotidiano das equipes de enfermagem. A busca foi realizada em quatro bases de dados, aplicando os filtros "texto completo", publicações a partir de 2012. Após o refinamento, foram obtidos 27 estudos para análise. Do total, sete citaram a dor lombar como manifestação mais significativa na equipe de enfermagem, variando com prevalência de 21,2 a 75\%. Outras áreas corporais citadas foram: braços e punhos; pescoço; ombros; pé/tornozelo; costas, pescoço e ombro esquerdo. Os fatores de risco para os distúrbios musculoesqueléticos encontrados estão relacionados ao tipo de trabalho exercido, setor de trabalho, às longas e exaustivas jornadas de trabalho, à sobrecarga de trabalho, postura inadequada entre outros. Fica evidente a necessidade de implantação de programas de saúde que visam a promoção da saúde e prevenção de agravos, além de monitoramento dos trabalhadores quanto à saúde física e mental, com vistas à melhora da qualidade de vida no trabalho.

ABSTRACT. The professional, when performing a work, exposes itself to the risks related to it, being able to physically and mentally ill, due to the changes in the work processes, associated with the intense rhythm and long hours. Workers in the nursing team, specifically, provide direct assistance to the individual over long periods of time, which increases the exposure to occupational hazards. Integrative review of national and international literature whose objective was to identify the ergonomic 
risks in the daily routine of nursing teams. The search was performed in four databases, applying the "full text" filters, publications from 2012. After refinement, 27 studies were obtained for analysis. Of the total, seven cited low back pain as the most significant manifestation in the nursing team, varying with a prevalence of 21.2 to $75 \%$. Other corporal areas mentioned were: arms and wrists; neck; shoulders; foot / ankle; back, neck and left shoulder. The risk factors for musculoskeletal disorders are related to the type of work performed, the work sector, the long and exhaustive working days, the overload of work, inadequate posture among others. It is evident the need to implement health programs aimed at health promotion and prevention of injuries, as well as monitoring of workers regarding physical and mental health, with a view to improving the quality of life at work.

\section{Introdução}

A Revolução Industrial proporcionou destaque na evolução das relações de trabalho, resultante do desenvolvimento acelerado, aliado às conquistas sociais de igualdade e liberdade. Assim surgiram várias dificuldades para a concretização dos direitos sociais no período pré-evolução tecno-científica, uma vez presentes os efeitos da globalização e a escassez de recursos naturais. Emergindo então a regulamentação jurídica e a garantia dos direitos pelo Estado (STURZA; MARQUES, 2017). Assim sendo, o trabalho passa a ser considerado um direito social, estabelecido por lei, como prática livre, desde que atendidas competências profissionais exigidas pela legislação (BRASIL, 1988).

É bem verdade existir ponto de conflito com a essência humana, algo natural proveniente de uma relação social determinada pela história, em transformação (HEIDEMANN; TOLEDO; BOECHAT, 2014). No entanto, constitui-se necessidade básica do ser humano, ao permitir alcançar outras esferas da vida e proporcionar satisfação, visto que os valores sociais objetivam generalizar grupos específicos de necessidades. O trabalho transforma o homem à medida que se faz produzir (STURZA; MARQUES, 2017).

Ao desempenhar um trabalho o homem está submetido a riscos ocupacionais, classificados em: físicos, químicos, biológicos, de acidentes e ergonômicos. De acordo com a classificação do Ministério do Trabalho, os riscos físicos são representados pela cor verde e dizem respeito às diversas formas de energia, tais como ruídos, radiações, frio, umidade e pressão anormal. Aqueles denominados como químicos, e simbolizados pela cor vermelha, levam em consideração as substâncias, produtos ou compostos que podem infiltrar no organismo, como gases, fumos, neblinas, poeiras, dentre outros. O biológico é caracterizado pela cor marrom, envolvem microrganismos como vírus, fungos, bactérias etc. Os de acidente são identificados pelo azul, se relacionam com situações que interferem na integridade do trabalhador. Por exemplo, arranjo físico inadequado, eletricidade, animais peçonhentos, máquinas sem proteção etc. E o ergonômico, tema aqui estudado, padronizado na cor amarela, tido como fatores que afetam características fisiológicas e 
psicológicas do homem, como por exemplo, repetitividade, postura inadequada e ritmo excessivo de trabalho (BRASIL, 1978; BRASIL, 1994).

O trabalhador, exposto aos riscos acima descritos, pode adoecer física e mentalmente, devido às mudanças nos processos de trabalho, associadas ao ritmo intenso e longas jornadas de trabalho. Como consequência caminha-se para pior qualidade de vida e, até mesmo, à incapacidade definitiva para o retorno às atividades (ANTUNES; PRAUN, 2015). Portanto, o objetivo do presente estudo foi identificar na literatura científica os riscos ergonômicos no cotidiano das equipes de enfermagem.

\section{Fundamentação}

Do ponto de vista epidemiológico, em nível mundial ocorrem 337 milhões de acidentes de trabalho não-mortais a cada ano. Doenças relacionadas ao trabalho atingem o quantitativo de 160 milhões de novos casos. E mortes ligadas aos acidentes 358 mil episódios e às doenças ocupacionais, os números somam 1,96 milhão. (OIT, 2014). No Brasil, em 2015, cerca de 500 mil acidentes (82\%) foram comunicados oficialmente (BRASIL, 2015).

Muito se tem discutido sobre a saúde no âmbito do trabalho, com destaque para os temas relacionados a equipamentos utilizados, divisão do trabalho e ergonomia, com vistas à qualidade de vida do trabalhador (MARINHO; ALMEIDA; ANDRADE, 2015).

Há suposições que, desde a pré-história o homem se preocupava com o manuseio dos objetos construídos a partir da pedra, sendo que os indícios se baseiam no formato padrão das ferramentas da época, adequados às características dos trabalhadores. Mas, a Ergonomia como uma disciplina científica, surge na década de 40. Resulta da evolução das tecnologias que se apresentavam como problema para as pessoas, para o manuseio de equipamentos. A relação entre ambiente, equipamentos e pessoas passa a ser estudada enquanto ciência do trabalho, que visa analisar a harmonia entre ser humano e o trabalho (CORRÊA; BOLETTI, 2015).

No que tange aos profissionais da área da saúde há alta carga de trabalho e a intensidade do trabalho levam a manifestações de ordem mental ou física (FILHO, 2015). Os trabalhadores da equipe de enfermagem, especificamente, prestam assistência direta ao indivíduo, em longos períodos de tempo no ambiente de trabalho, o que aumenta a exposição aos riscos ocupacionais (MARINHO; ALMEIDA; ANDRADE, 2015). Nos serviços de emergência, por exemplo, tal situação gera um efeito de estresse nos trabalhadores, uma vez que a assistência deve ser imediata, ágil e eficaz, o que consequentemente afeta a saúde física e mental dos profissionais (LORO; ZEITOUNE, 2017). 
Tais riscos e suas consequências à saúde do trabalhador são um problema de saúde pública, interferem no desempenho do trabalho executado e, principalmente, na qualidade de vida humana. Os resultados do estudo poderão auxiliar gestores, profissionais de saúde e serviços de saúde a direcionar ações para controlar os riscos ergonômicos e a reduzir a incidência de agravos/doenças relacionadas ao trabalho, bem como caminhar para a qualidade de vida do trabalhador.

\section{Método}

Trata-se de uma revisão integrativa de literatura nacional e internacional sobre os riscos ergonômicos no cotidiano das equipes de enfermagem.

A busca de publicações foi realizada na Biblioteca Virtual em Saúde (BVS), que retornou estudos das bases de dados: Literatura Latino-americana e do Caribe em Ciências da Saúde (LILACS), Base de Dados de Enfermagem (BDENF), Medical Literature Analysis and Retrieval System Online MEDLINE) e Índice Bibliográfico Espanhol de Ciências da Saúde (IBECS).

Os descritores utilizados foram: "saúde do trabalhador", "engenharia humana", "riscos ocupacionais", "enfermagem". Foram incluídos artigos, publicados na íntegra, entre os anos de 2012 e 2017. A escolha do recorte temporal se fez devido ao ano de criação da Política Nacional de Saúde do Trabalhador e da Trabalhadora, em 2012.

A população constituiu-se de 495 artigos. Após a leitura de títulos foram selecionados 83 e depois da leitura de resumos, 59 trabalhos. Considerando o tema proposto, foram obtidos 27 estudos para análise (Quadro 1).

\section{Resultados e discussão}

Dos 27 estudos que compuseram a amostra, 12 (44,4\%) foram publicados na MEDLINE, nove (33,3\%) na LILACS, cinco (18,51\%) na BDENF e um $(3,7 \%)$ na IBECS. Do total encontrado, $16(59,2 \%)$ artigos foram escritos em inglês, nove (33,3\%) em língua portuguesa e dois $(7,4 \%)$ em espanhol.

A dor lombar foi mencionada em sete (25,9\%) dos 27 estudos, presente na equipe de enfermagem com variação de $21,2 \%$ a $75,5 \%$, sendo identificados como fatores relacionados: postura inadequada, longos períodos de trabalho na mesma posição, sobrecarga de trabalho, tarefas repetitivas (ALPEROVITCH-NAJENSON et al, 2015; ATTAR, 2014; GHILAN et al, 2013; PETERSEN; MARZIALE, 2014; SHIEH et al, 2016; SHOJl et al, 2016; WARNAKULASURIYA et al, 2012). 
Quadro 1 - Resultados do cruzamento entre descritores sobre os riscos ergonômicos nas equipes de enfermagem no período 2012-2017 - Belo Horizonte, 2017

\begin{tabular}{|l|c|c|c|c|c|}
\hline \multicolumn{1}{|c|}{$\begin{array}{c}\text { Cruzamento dos } \\
\text { Descritores }\end{array}$} & $\begin{array}{c}\text { Total de } \\
\text { artigos sem } \\
\text { filtros }\end{array}$ & $\begin{array}{c}\text { Total de } \\
\text { artigos com } \\
\text { filtros }\end{array}$ & $\begin{array}{c}\text { Leitura } \\
\text { de } \\
\text { título }\end{array}$ & $\begin{array}{c}\text { Leitura } \\
\text { de } \\
\text { resumos }\end{array}$ & $\begin{array}{c}\text { Leitura } \\
\text { na } \\
\text { íntegra }\end{array}$ \\
\hline $\begin{array}{l}\text { Enfermagem x Saúde do } \\
\text { Trabalhador x Engenharia } \\
\text { Humana x Riscos Ocupa- } \\
\text { cionais }\end{array}$ & 66 & 3 & 2 & 1 & 1 \\
\hline $\begin{array}{l}\text { Enfermagem x Saúde do } \\
\text { Trabalhador x Engenharia } \\
\text { Humana }\end{array}$ & 277 & 14 & 7 & 4 & 1 \\
\hline $\begin{array}{l}\text { Enfermagem x Saúde do } \\
\text { Trabalhador x Riscos Ocu- } \\
\text { pacionais }\end{array}$ & 820 & 172 & 21 & 17 & 11 \\
\hline $\begin{array}{l}\text { Enfermagem x Engenharia } \\
\text { Humana x Riscos Ocupa- } \\
\text { cionais }\end{array}$ & 23 & 7 & 4 & 3 & 1 \\
\hline $\begin{array}{l}\text { Enfermagem x Riscos } \\
\text { Ocupacionais }\end{array}$ & 1.688 & 299 & 49 & 34 & 13 \\
\hline
\end{tabular}

Fonte: Os autores (2017)

A lombalgia foi evidenciada como principal manifestação decorrente das condições do trabalho, afetando respectivamente $59 \%$ e $29,4 \%$ da equipe de enfermagem (SILVA et al, 2016; SOUZA et al, 2015).

Um estudo realizado em um hospital do Piauí encontrou resultados semelhantes em relação a prevalência de sintomas osteomusculares: $88.3 \%$ dos trabalhadores de enfermagem apresentaram algum tipo de desconforto osteomuscular ao longo de doze meses, enquanto 58,3\% apesentaram desconfortos nos últimos sete dias (PACHECO et al, 2016).

O desconforto musculoesquelético foi um dos problemas ergonômicos mais prevalentes, mostrando que $85 \%$ da equipe de enfermagem apresentaram problemas relacionados a ergonomia, enquanto em outro estudo, foi apresentado um percentual de 75\% (ATTAR, 2014; NERY et al, 2013). Ambos estudos se referem ao corte de 12 meses para surgimento de queixas ou sintomas relacionados a riscos ergonômicos. Outros autores 
mostram que $57.7 \%$ da equipe apresentou algum sinal no prazo de 3 meses de exposição (GONZÁLEZ et al, 2014).

As lesões mais comuns encontradas no trabalho das equipes de enfermagem são de ordem musculoesqueléticas (71\%) (SMITH, 2012). Já em outro artigo, 49,5\% da equipe de enfermagem são afetados pelos distúrbios musculoesqueléticos (PRIETO; MÚNERA; LÓPEZ, 2015). Outros autores encontraram o total de $91,81 \%$ de profissionais da enfermagem com sintomas de tais distúrbios (VIDOR et al, 2014).

Essas alterações, que podem ser decorrentes de fatores psicossociais (GOLABADI et al, 2013), têm sido relatados como aspectos relevantes no desenvolvimento ou agravamento dos distúrbios musculoesqueléticos (OLIVEIRA; ALMEIDA, 2017), sendo destacado também que enfermeiros que trabalham mais de 10 horas diárias apresentaram risco maior de desenvolver doenças osteomusculares (ATTAR, 2014), uma vez que o trabalho executado de forma prolongada associado à postura incorreta e alta carga de atividades a serem realizadas podem contribuir para esses distúrbios.

A prevalência de dor lombar aumentou proporcionalmente à idade, sendo 65,2\% em enfermeiras com idade entre 20 e 24 anos e 75,5\% nos profissionais com 35 anos ou mais (SHIEH et al, 2016). Supõe-se que esse fato se relaciona aos prováveis longos anos de profissão e às condições fisiológicas que contribuem para o desenvolvimento da dor lombar nos indivíduos.

Um estudo que buscou investigar a prevalência de dor lombar entre enfermeiras no Yemen, constatou que 139 profissionais entrevistadas se ausentaram do trabalho devido à dor lombar (GHILAN et al, 2013). Ainda no âmbito do absenteísmo, encontrou-se um total de 144 casos notificados relacionados à carga de trabalho, das quais $20,7 \%$ corresponderam às doenças do sistema musculoesquelético e do tecido conjuntivo, representando 325 dias de afastamento do trabalho (MININEL et al, 2013).

Estudo semelhante divulgou que $19,7 \%$ dos atestados tiveram como justificativa as doenças do sistema osteomuscular e do tecido conjuntivo, com o total de 310 atestados, e em seguida os transtornos mentais e comportamentais, totalizando 284 atestados (18,04\%). Além disso, os profissionais que mais se ausentaram do trabalho respaldados pelos atestados médicos foram os técnicos e auxiliares de enfermagem, sendo que representam $81 \%$ da equipe de enfermagem, o que pode acabar gerando sobrecarga em outros profissionais e afetando a assistência prestada (MARQUES et al, 2015).

A maior taxa de dor lombar se fez presente nas profissões de enfermagem e em operadores de computadores (WARNAKULASURIYA et al, 2016), relacionada ao fato da permanência na mesma posição por prolongadas horas e ao tipo de trabalho executado que contribui para a ocorrência de dor lombar. O profissional de enfermagem também é destacado como uma das categorias que mais necessitam de prevenção de doenças osteomusculares, principalmente em relação à dor lombar (THIEDE et al, 2014). 
Ao estudar fatores de riscos físicos e psicossociais para distúrbios musculoesqueléticos entre enfermeiros do Brasil e da Itália, os autores verificaram na amostra estudada que existiu um risco maior de dor no pescoço e no ombro em profissionais que trabalharam com mãos acima do ombro por pelo menos uma hora diária (CARUGNO et al, 2012). Pode-se observar que os distúrbios osteomusculares afetam com variedade diversas regiões corporais, sendo este fato relacionado diretamente ao tipo de atividade exercido, tempo de trabalho etc.

Outros fatores relacionados aos distúrbios osteomusculares foram identificados, como: acúmulo de trabalho (WORM et al, 2016); posturas desconfortáveis (GONZÁLEZ et al, 2014; THIEDE et al, 2014); repetitividade de movimentos, jornadas exaustivas, sobrecarga de trabalho (GONZÁLEZ et al, 2014; GOUVEIA; OLIVEIRA; LIRA, 2016); posições desconfortáveis relacionadas à transferência de pacientes (THIEDE et al, 2014) e mudanças de posição do paciente no leito/banho/chuveiro (LEMO et al, 2012) mudanças forçadas e antigravitacionais dos membros superiores (PRIETO; MÚNERA; LÓPEZ, 2015); cargas intensas de trabalho e ambiente físico na ocorrência de estresse (YADA et al, 2014); obesidade, fraca saúde física, características do trabalho (REED et al, 2014). Os profissionais de enfermagem trabalham diretamente realizando movimentações/transferências, com excesso de carga horária, posturas viciosas e incorretas, uso de calçados inadequados. Tais situações são consideradas fatores de risco para o principal sintoma osteomuscular: a dor (SILVA et al, 2016).

Problemas de ordem circulatória de membros inferiores foram observados: varizes relacionadas à posição monótona durante extensas horas de trabalho, sem pausas ou período de descanso (BELEZA et al, 2013; SHOJI; SOUZA; FARIAS, 2015). Uma pesquisa que visou avaliar a prevalência de fatores de riscos para varizes em profissionais que trabalham prolongadamente de forma ortostática, pontuou que mais da metade $(51 \%)$ da amostra, portadores de varizes afirmaram trabalhar em média 8 horas na posição ortostática, fato que condiz com o cenário de trabalho dos profissionais de enfermagem (FERRAZ et al, 2015).

Outras partes do corpo foram citadas como locais afetados por diversos fatores do cotidiano profissional. Respectivamente, são elas: braços e punhos; pescoço; ombros; pé/tornozelo; costas, pescoço e ombro esquerdo (ALPEROVITCH-NAJENSON et al, 2014; GONZÁLEZ et al, 2014; NERY et al, 2013; PRIETO; MÚNERA; LÓPEZ, 2015; REED et al, 2014). Os ombros foram a região mais acometida por uma alta taxa de distúrbios osteomusculares nos 12 meses anteriores ao estudo, com prevalência de 40\%, assim como os joelhos (33,3\%) e região cervical (28,6\%) (SILVA et al, 2016). Também foi destacada a região cervical como a segunda parte do corpo mais afetada pela dor osteomuscular $(20,7 \%)$, seguida da parte dorsal $(10,9 \%)$, joelhos $(5,4 \%)$, coxas $(1 \%)($ SOUZA et al, 2015$)$. 
Em relação às lesões nos braços, estão relacionadas às atividades cansativas e de levantamento de peso (FONTANA; LAUTERT, 2013). Concomitante a isso, $60 \%$ dos enfermeiros participantes do estudo que objetivou identificar os fatores de risco aos quais o enfermeiro intensivista está exposto, relataram que o uso dos braços de forma contínua é uma ação bastante exigida no Centro de Terapia Intensiva em que trabalham (ALBUQUERQUE et al, 2015), podendo ocasionar lesões e contribuir para o absenteísmo do local de trabalho.

Além disso, foi ressaltado que as profissões que mais sofrem com o estresse envolvem enfermeiras educadoras, enfermeiras psiquiátricas, parteiras registradas e auxiliares de enfermagem (SMITH, 2012), sendo que essa condição também foi um ponto evidenciado como fator de influência para o desenvolvimento da Síndrome de Burnout (SB) (CARVALHO; MAGALHÃES, 2013; SANCHEZ; OLIVEIRA, 2016). Associado ao estresse, outros fatores também contribuem: aspectos do ambiente de trabalho, falta de expectativa profissional, sobrecarga física e mental e insatisfação salarial (CUNHA; SOUZA; MELLO, 2012).

Em uma pesquisa sobre as dimensões sintomatológicas da SB foi encontrado que uma parte relevante dos profissionais da enfermagem apresentou sintomas da síndrome, sendo que $85,2 \%$ manifestaram exaustão emocional e 96,3\% despersonalização (OLIVEIRA; ARAÚJO, 2016).

As medidas preventivas se fazem, portanto, por meio de avaliação ergonômica do trabalho executado e identificar riscos, bem como propor intervenções (MARQUES et al, 2015), analisar o ambiente social/físico e aspectos de organização e características das atividades, com medidas práticas como pausas ao longo do dia, revisão da produtividade, alternância de serviços realizados etc. (PAULA; SANCHEZ; PEREIRA, 2016).

\section{Considerações finais}

O estudo permitiu concluir que os riscos ergonômicos estão presentes nas mais diversas profissões, principalmente no ambiente de trabalho dos componentes das equipes de enfermagem. Foram identificados riscos variados relacionados à ergonomia, desde somente o desconforto osteomuscular até a manifestação de dor lombar e varizes.

Pode-se perceber também que tais riscos são diretamente proporcionais ao tipo de trabalho exercido, setor de trabalho, às longas e exaustivas jornadas de trabalho, à sobrecarga de trabalho, postura inadequada etc.

Fica evidente a necessidade de implantação/reforço de serviços de ginástica laboral e acompanhamento dos trabalhadores quanto à saúde física e mental, com vistas à melhora da qualidade de vida no trabalho, uma vez que a satisfação e valorização do trabalhador contribui para o melhor rendimento deste e, consequentemente, para o alcance dos 
resultados esperados e das metas pactuadas, por parte da instituição à qual o profissional é vinculado.

Portanto, se faz necessário também avaliar o ambiente de trabalho quanto às condições ergonômicas, identificando riscos e propondo intervenções, revisar o processo de trabalho e atividades que são exercidas, para evitar adoecimentos causados pelo trabalho e reduzir o índice de absenteísmo.

\section{Referências}

ALBUQUERQUE, Saemmy Grasiely Estrela; CASTRO, Ricardo Dias; FERREIRA, Gabriela Lacet Silva; OLIVEIRA, Karla de Lima. Fatores de Risco à Segurança do Enfermeiro na Unidade de Terapia Intensiva de um Hospital Geral. Rev. bras. ciênc. saúde; 19(2): 135142, 2015.

ALPEROVITCH-NAJENSON, Deborah; SHEFFER, Dvora; TREGER, luly; FINKELS, Tova; KALICHMAN, Leonid. Rehabilitation versus Nursing Home Nurses' Low Back and NeckShoulder Complaints. Rehabilitation Nursing 2015, 40, 286-293.

ANTUNES, Ricardo; PRAUN, Luci. A sociedade dos adoecimentos no trabalho. Serv. Soc. Soc., São Paulo, n. 123, p. 407-427, jul./set. 2015.

ATTAR, Suzan. Frequency and risk factors of musculoskeletal pain in nurses at a tertiary centre in Jeddah, Saudi Arabia: a cross sectional study. BMC Res Notes; 7: 61, 2014 Jan 25.

BELEZA, Cinara Maria Feitosa; GOUVEIA, Márcia Teles de Oliveira; ROBAZZI, Maria Lúcia do Carmo Cruz Robazzi; TORRES, Cynthia Roberta Dias. Riesgos ocupacionales y problemas de salud percibidos por trabajadores de enfermería en una unidad hospitalaria. Cienc. enferm. vol.19 no.3 Concepción 2013.

BRASIL, 1978. NR 9 - Programa de Prevenção de Riscos Ambientais. Disponível em: http://trabalho.gov.br/images/Documentos/SST/NR/NR09/NR-09-2016.pdf Acesso em: 09 de nov. 2017.

BRASIL, 1988. Constituição da República Federativa do Brasil de 1988. Disponível em: http://www.planalto.gov.br/ccivil 03/constituicao/constituicao.htm Acesso em 10 de nov. 2017.

BRASIL, 1994. Portaria n.o 25, de 29 de dezembro de 1994. Disponível em: https://www.agencia.cnptia.embrapa.br/Repositorio/Portaria+n.+25+SSST+MTb+29+dezemb ro+1994+Aprova+a+NR+9+sobre+o+Programa+de+Prevencao+e+riscos+ambientais 000gv pl14yq02wx7ha0g934vgrnn5ero.PDF Acesso em: 09 de nov. 2017. 
BRASIL, 2015. Anuário Estatísticos de Acidentes do Trabalho. Disponível em: http://www.previdencia.gov.br/wp-content/uploads/2017/05/aeat15.pdf Acesso em 11 de nov. 2017.

CARUGNO, Michele; PESATORI, Angela Cecilia; FERRARIO, Marco Mario; FERRARI, Andrea Lepos; SILVA, Fabio Jose; MARTINS, Aline Caldas; FELLI, Vanda Elisa Andres; COGGON, David; BONZINI, Matteo. Physical and psychosocial risk factors for musculoskeletal disorders in Brazilian and Italian nurses. Cad Saude Publica; 28(9): 1632-42, 2012 Sep.

CARVALHO, Clecilene Gomes; MAGALHÃES, Sérgio Ricardo. Who cares for the caregiver: key factors that affect the health of nursing Professionals, a biopsychosocial view. Rev. pesqui. cuid. fundam. (Online); 5(3): 122-131, jul.-set. 2013. CÔRREA, Vanderlei Moraes; BOLETTI, Rosane Rosner. Ergonomia: Fundamentos e Aplicações. Porto Alegre: Bookman, 2015.

CUNHA, Ana Paula; SOUZA, Emanuelle Marques; MELLO, Rosâne. Os fatores intrínsecos ao ambiente de trabalho como contribuintes da síndrome de Burnout em profissionais de enfermagem. Rev. pesqui. cuid. fundam. (Online); 4(supl.1): 29-32, 2012.

FERRAZ, Renato Ribeiro Nogueira; MACEDO, Rosiane Souza; FORNARI, João Vitor; BARBABÉ, Anderson Sena. Prevalência de varizes em trabalhadores mantidos em posição ortostática. Rev. Bras. Pesq. Saúde, Vitória, 17(3): 6-13, jul-set, 2015.

FILHO, José Marçal Jackson. Engajamento no trabalho, impedimentos organizacionais e adoecer: a contribuição da Ergonomia da Atividade no setor público brasileiro. Rev. bras. saúde ocup. vol.40 no.131 São Paulo Jan./June 2015.

FONTANA, Rosane Teresinha; LAUTERT, Liana. The situation of nursing work and occupational risks from an ergological perspective. Rev Lat Am Enfermagem; 21(6): 1306-1313, Nov-Dec/2013.

GHILAN, Khaled; AL-TAIAR, Abdulla; YOUSFI, Nuha AI. ZUBAIDI, Rania Al; AWADH, Iman; AL-OBEYED, Zaher. Low back pain among female nurses in Yemen. Int $\mathrm{J}$ Occup Med Environ Health; 26(4): 605-14, 2013 Aug.

GOLABADI, Majid; ATTARCHI, Mirsaeed; RAEISI, Saeed; NAMVAR, Mohamad. Effects of psychosocial strain on back symptoms in Tehran general hospital nursing personnel. Arh Hig Rada Toksikol 2013;64:505-512.

GONZÁLEZ, Manuel Fernández; VALENCIA, Mónica Fernández; HUERTA, María Ángeles Manso; RODRíGUEZ, Maria Paz Gómez; RECIO, Maria Carmen Jiménez; DÍAZ, Faustino delCoz. Transtornos musculoesqueléticos en personal auxiliar de enfermería del Centro Polivalente de Recursos para Personas Mayores "Mixta" de Gijón - C.P.R.P.M. Mixta. Gerokomos vol.25 no.1 Barcelona mar. 2014. 
GOUVEIA, Márcia Teles de Oliveira; OLIVEIRA, Vanessa Cavalcante; LIRA, Ivana Mayra da Silva. Riscos ergonômicos em um Centro de Material e Esterilização. Rev. enferm. UFPI; 5(3): 42-47, jul.-set.2016.

HEIDEMANN, Heinz Dieter; TOLEDO, Carlos de Almeida; BOECHAT, Cássio Arruda. 0 trabalho no Brasil: traçado interpretativo de sua história de formação e de sua crítica. Estud. av. vol.28 no.81 São Paulo May/Aug. 2014.

LEMO, A; SILVA, AG; TUCHERMAN, M; TALERMAN, C; GUASTELLI, RL; BORBA, CL. Risk reduction in musculoskeletal practice assistance professional nursing pilot in semi intensive care unit. Work; 41 Suppl 1: 1869-72, 2012.

LORO, Marli Maria; ZEITOUNE, Regina Célia Gollner. Collective strategy for facing occupational risks of a nursing team. Rev. esc. enferm. USP vol.51. São Paulo 2017. Epub Mar 09, 2017.

MARINHO, Maykon dos Santos; ALMEIDA, Camila Tambone; ANDRADE, Everaldo Nery. Risco ergonômico nas práticas da equipe de enfermagem de uma UTI. C\&D-Revista Eletrônica da Fainor, Vitória da Conquista, v.8, n.1, p.192-205, jan./jun. 2015.

MARQUES, Divina de Oliveira; PEREIRA, Milca Severino; SOUZA, Adenícia Custódio Silva; VILA, Vanessa da Silva Carvalho; ALMEIDA, Carlos Cristiano Oliveira de Faria; OLIVEIRA, Enio Chaves. O absenteísmo - doença da equipe de enfermagem de um hospital universitário. Rev Bras Enferm. 2015 set-out;68(5):876-82.

MININEL, Vivian Aline; FELLI, Vanda Elisa Andres; SILVA, Everaldo Jose; TORRI, Zelinda; ABREU, Ana Paula; BRANCO, Maria Tereza Afonso. Workloads, strain processes and sickness absenteeism in nursing. Rev Lat Am Enfermagem; 21(6): 1290-1297, NovDec/2013.

NERY, Denise; TOLEDO, Aline Martins; JÚNIOR, Silvio Oliveira; TACIRO, Charles; CARREGARO, Rodrigo. Análise de parâmetros funcionais relacionados aos fatores de risco ocupacionais da atividade de enfermeiros de UTI. Fisioter. Pesqui. vol.20 no.1 São Paulo mar. 2013.

OIT, 2014. Trabalho só pode ser decente se for seguro e saudável. Disponível em: http://www.oit.org.br/content/trabalho-so-pode-ser-decente-se-seguro-e-saudavel Acesso em 12 de nov. 2017.

OLIVEIRA, Liliane Pereira Santos; ARAÚJO, Giovana Fernandes. Características da Síndrome de Burnout em enfermeiros da emergência de um hospital público. Revista Enfermagem Contemporânea. 2016 Jan./Jun.;5(1):34-42.

OLIVEIRA, Vanessa Cotian; ALMEIDA, Rogério José. Aspectos que Determinam as Doenças Osteomusculares em Profissionais de Enfermagem e seus Impactos Psicossociais. J Health Sci 2017;19(2):130-5. 
PACHECO, Edildete Sene; SOUZA, Agna Roberta Rodrigues; SOUSA, Pollyana Taiana de Morais; ROCHA, Adriene Fonseca. Prevalence of musculoskeletal symptoms related to nursing work in the hospital field. Rev Enferm UFPI. 2016 Oct-Dec;5(4):31-7.

PAULA, Ana de Assis; SANCHEZ, Maritza Ortiz; PEREIRA, Marilia. Lesões por Esforço Repetitivo/Distúrbio Osteomuscular Relacionado ao Trabalho: contribuições para a prevenção em trabalhadores de enfermagem Repetitive Strain Injuries/Musculoskeletal Disorders. ACC CIETNA. Año 4. № 1 pp. (7-20) 2016.

PETERSEN, Rafael de Souza; MAZIALE, Maria Helena Palucci. Low back pain characterized by muscle resistance and occupational factors associated with nursing. Rev Lat Am Enfermagem; 22(3): 386-393, May-Jun/2014.

PRIETO, Amparo Astrid Montalvo; MÚNERA, Yesica María Cortés; LÓPEZ, Martha Cecilia Rojas. Riesgo ergonómico asociado a sintomatología musculoesquelética en personal de enfermería. Hacia promoc. salud; 20(2): 132-146, jul.-dic. 2015.

REED, Lloyd; BATTISTUTTA, Diana; YOUNG, Jeanine; NEWMAN, Beth. Prevalence and risk factors for foot and ankle musculoskeletal disorders experienced by nurses. BMC Musculoskelet Disord; 15: 196, 2014 Jun 05.

SANCHEZ, Fernanda Ferreira Santiago; OLIVEIRA, Reginaldo. Aspectos mediadores e desencadeadores da síndrome de burnout nos enfermeiros. CuidArte, Enferm; 10(1): 61-67, jan.-jun. 2016.

SHIEH, Shwn-Huey; SUNG, Fung-Chang; SU, Chia-Hsien; TSAI, Yafang; HSIEH, Vivian Chia-Rong. Increased low back pain risk in nurses with high workload for patient care: A questionnaire survey. Taiwan J Obstet Gynecol; 55(4): 525-9, 2016 Aug.

SHOJI, Shino; SOUZA, Norma Valéria Dantas de Oliveira; FARIAS, Sheila Nascimento Pereira. Impacto do ambiente laboral no processo saúde doença dos trabalhadores de enfermagem de uma unidade ambulatorial especializada. REME rev. min. enferm; 19(1): 43-48, jan.-mar. 2015.

SHOJI, Shino; SOUZA, Norma Valéria Dantas de Oliveira; FARIAS Sheila Nascimento Pereira; VIEIRA, Manoel Luíz Cardoso; PROGIANTI, Jane Marcia. Proposta de melhoria das condições de trabalho em uma unidade ambulatorial: perspectiva da enfermagem. Esc. Anna Nery Rev. Enferm; 20(2): 303-309, abr.-jun. 2016.

SILVA, Isneander; ALVES, Natan; NOGUEIRA, Mariane; MENDONÇA, Rafael; ALVES, Fernanda; ALVES, Aleandro; VALENTE, Pedro; ARAÚJO, Thayza; SOUZA, Eduardo; CUNHA, Renata; PIRES, Marília; FANTINATI, Marcelo. Incidência dos sintomas osteomusculares relacionados ao trabalho da equipe de enfermagem do Hospital Santa Gemma/AFMBS. Revista Faculdade Montes Belos (FMB), v. 9, n², 2016, p (28141), 2014.

SMITH, Elizabeth. How are nurses at risk?. Work; 41 Suppl 1: 1911-9, 2012. 
SOUSA, Milena Nunes Alves de; SILVA, Genildo Medeiros; COSTA, Tarciana Sampaio; NUNES, Rosa Martha Ventura; MEDEIROS, Hellen Renatta Leopoldo. Prevalência de distúrbios osteomusculares em enfermeiros. FIEP BULLETIN - Volume 85 - Special Edition - ARTICLE I- 2015.

STURZA, Janaína Machado; MARQUES, Aline Damian. A Importância do Trabalho para a Consolidação da Dignidade do Homem: Apontamentos sob a Perspectiva dos Direitos Sociais. Direito, Estado e Sociedade, n.50 p. 109 a 125 jan/jun 2017.

THIEDE, Markus; LIEBERS, Falk; SEIDLER, Andreas; GRAVEMEYER, Stefan; LATZA, Ute. Gender specific analysis of occupational diseases of the low back caused by carrying, lifting or extreme trunk flexion--use of a prevention index to identify occupations with high prevention needs. Am J Ind Med; 57(2): 233-44, 2014 Feb.

VIDOR, Cristiane da Rocha; MAHMUD, Mahmud Ahmad Ismail; FARIAS, Leonardo Fontanive; SILVA, César Augusto; FERRARI, Juliana Nery; COMEL, João Carlos; ZANINI, Maurice; NERY, Rosane Maria; SANTOS, Antônio Cardoso; STEFANI, Marco Antônio. Prevalência de dor osteomuscular em profissionais de enfermagem de equipes de cirurgia em um hospital universitário. Acta Fisiatr. 2014;21(1):6-10 WARNAKULASURIYA, SSP; PEIRIS-JOHN, RJ; COGGON, D; NTANI, G; SATHIAKUMAR, $\mathrm{N}$; WICKREMASINGHE, AR. Musculoskeletal pain in four occupational populations in Sri Lanka. Occup Med (Lond); 62(4): 269-72, 2012 Jun.

WORM, Fabiana Adol; PINTO, Márcia Aparecida Oliveira; SCHIAVENATO, Diego; ASCARI, Rosana Amora; TRINDADE, Letícia de Lima; SILVA, Olvani Martins. Risco de adoecimento dos profissionais de enfermagem no trabalho em atendimento móvel de urgência. Rev. cuid. (Bucaramanga. 2010); 7(2): 1288-1296, july.-dic. 2016.

YADA, Hironori; ABE, Hiroshi; LU, Xi; WAKIZAKI, Yuko; OMORI, Hisamitsu; MATSUO, Hisae; ISHIDA, Yasushi; KATOH, Takahiko. Job-related stress in psychiatric nurses in Japan caring for elderly patients with dementia. Environ Health Prev Med. 2014 Nov; 19(6): 436-443. 